\title{
Recurrent endobronchial fibro-epithelial polyposis in a 73-year-old woman
}

\author{
Ramin Sami $^{\circledR}$, Mina Nickpour ${ }^{1 *}$, Noushin Afshar Moghaddam ${ }^{(\mathbb{}}$ \\ ${ }^{1}$ Department of Internal Medicine, Isfahan University of Medical Sciences, Isfahan, Iran \\ ${ }^{2}$ Department of Pathology, Shahid Beheshti University of Medical Sciences, Tehran, Iran
}

\author{
*Correspondence to \\ Mina Nickpour, Email \\ drminanickpour@sbmu.ac.ir \\ Received 5 Jan. 2021 \\ Accepted 10 Feb. 2021 \\ Published online 20 Feb. 2021 \\ Keywords: Fibroepithelial \\ polyp, Bronchoscopy, \\ Immunohistochemistry, Benign \\ tumor
}

Citation: Sami R, Nickpour M, Afshar Moghaddam N Recurrent endobronchial fibro-epithelial polyposis in a 73-year-old woman. Immunopathol Persa. 2021;7(2):e14. DOI: 10.34172 ipp. 2021.14

\section{Abstract}

Introduction: Fibroepithelial polyp (FEP) is a benign tumor that is presented frequently in some of the organs like oral cavity, skin, colon and genitourinary tract, but in airway is rare.

Case Presentation: We present a rare case in this paper, with recurrent bronchial FEP that was near completely obstructed right main bronchus that we managed her successfully with interventional bronchoscopy. In this case report we describe this rare condition.

Conclusion: Airway FEP is uncommon benign tumor that may obstruct airways and rarely may be recurrent like our case.

\section{Introduction}

Benign tumors of the tracheobronchial tree are quite rare and consistute $\%$ of all lung tumors. Most frequent are hamartomas and papillomas and less frequent are lipomas, leiomyomas and neurogenic tumors (1).

FEP is one of the benign tumors in some organs like oral cavity, skin, colon and genitourinary tract and in some literature were reported in upper and lower airways as endobronchial lesions $(2,3)$. FEP is a smooth-surfaced polyp that is known as a mesenchymal tumor (4).

It seems to be difficult for clinicians to differentiate and correctly diagnosis these lesions to decide appropriate treatment while some of these patients for months or even years remains undiagnosed or was managed as asthma or chronic obstructive pulmonary disease (COPD). Histopathological interpretation helps us to distinguish this benign lesion from malignant lesions, because figure and radiographic manifestation of lesion are not specific (5).

\section{Case Presentation}

A 73-year-old woman with past medical history of diabetes mellitus type 2 and hypertension from 30 years ago was treated with oral agents and was controlled very well. She was housekeeper, non-smoker and did not have any pet and history of exposure to

\section{Key point}

Fibroepithelial polyp (FEP) is a benign tumor that is presented frequently in some of the organs like oral cavity, skin, colon and genitourinary tract, but in airway is rare and was not reported yet as a recurrent mass.

chemical substances or allergy and atopy and not recently taking new drugs.

She did not complain any respiratory symptoms until October 2017. She experienced shortness of breath that was gradually progressive. The patient suffered dyspnea that limited her usual activity. Our patient complained heart-burn and gastroesophageal reflux and dry cough that was exacerbated when was laying. There was no fever, weight loss or other symptoms. Laboratory test, electrocardiography, echocardiography and upper endoscopy were normal. Then she was referred to a pulmonologist. In examination, wheezing in right hemithorax was ausculated. No other abnormal findings were present. Spirometry was obstructive pattern. Lung HRCT scan demonstrated partial obstruction in right main bronchus and central tubular bronchiectasis that associated with peribronchial cuffing, with airway transmural calcification suggestive tracheopathia racemose. Right lower zone band like fibroatelectasis appearing consolidation was

Copyright $\odot 2021$ The Author(s); Published by Nickan Research Institute. This is an open-access article distributed under the terms of the Creative Commons Attribution License (http://creativecommons.org/licenses/by/4.0), which permits unrestricted use, distribution, and reproduction in any medium, provided the original work is properly cited. 
present.

Flexible bronchoscopy (FUJI, EB-530H) was performed. Vocal cord, trachea and left lung were normal, without any endobronchial mass. In right lung, a large endobronchial mass in right main bronchus was seen that near $70 \%$ whole of right bronchus was obstructed. The appearance of mass was smooth, shiny, bright and round shape, without any ulcer (Figure 1). Then for assurance of airways support and ventilation, rigid bronchoscopy was done. With electrocautery tumor destruction was performed and rigid forceps-aid, whole of tumor was resected. Hematoxylin and eosin stain of biopsies showed polypoid tissue was covered by respiratory type epithelium and contain fibrovascular stroma (Figure 2). The stromal component of fibroepithelial polyp (FEP) showed benign looking stromal cells without cytological atypia and mitosis (Figure 3). Immunohistochemistry study (IHC), pan-CK, CD34 and $\mathrm{S} 100$ were negative. Vimentin was positive and less than $1 \%$ stromal component had Ki-67. After one month follow-up visit, the patient was well without any complaint.

The patient was asymptomatic for more than 2 years.But from that time onward she began to experience dyspnea in exercise, the severity of dyspnea was gradually increased after few months. With suspicion of recurrence in previous mass, lung HRCT scan was conducted that showed filling defect in origin of right main bronchus with lobulated border measuring 34X15 mm (Figure 4). Flexible bronchoscopy demonstrated a large mass in right main bronchus exactly in previous site that obstructed whole of right bronchus. Origin of mass with a short pedicle was in intermedius bronchus. Then rigid bronchoscopy was done and the entire mass with forceps rigid and electrocautery was resected. Pathological and IHC study showed FEP like 2 years ago and confirmed the last diagnosis. After resection of tumor, the patient was symptom-free.

\section{Discussion}

FEP of airways is a rare benign tumor described in 1930 by Patterson that sometimes is misdiagnosed with other differential diagnosis like carcinoid, neurogenic tumor, leiomyoma, lipoma, hamartoma, papilloma and neoplasm (1).

Several chronic pro-inflammatory etiological factors like asthma, COPD, aspiration of foreign body, chronic sinusitis, tuberculosis, chronic chemical and smoke inhalation and prolonged mechanical ventilation are contributed to this situation (6).

Symptoms are variable. Some patients are asymptomatic while symptomatic patients have coughing, mild to severe dyspnea, recurrent pneumonia and hemoptysis (5).

Chest radiography sometimes is normal. However in some cases in CT, airway filling defect and postobstruction bronchiectasis and fibro atelectasis and consolidation are seen $(5,7)$

It consisted of fibrovascular stroma covered by normal respiratory epithelium (7). Benign stromal cells without

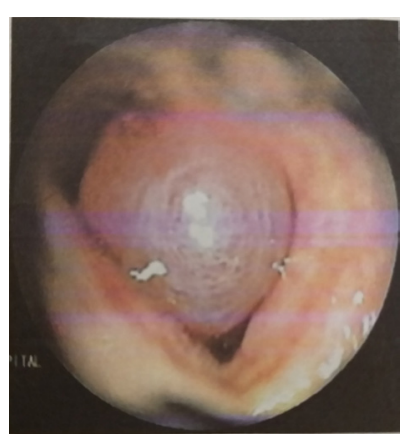

Figure 1. Endobronchial round and shiny mass in right main bronchus.

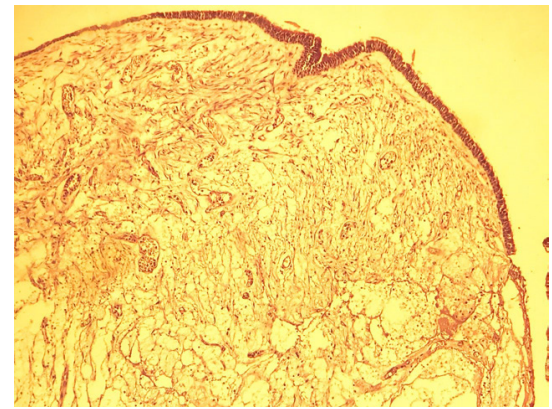

Figure 2. A polypoid tissue covered by respiratory type epithelium (H\&E staining).

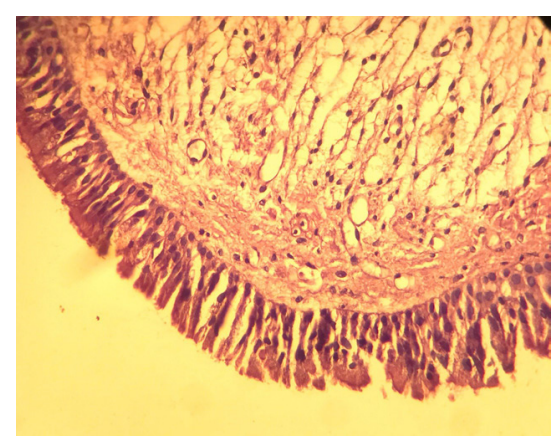

Figure 3. Fibroepithelial polyp respiratory type epithelium and fibrovascular stroma.

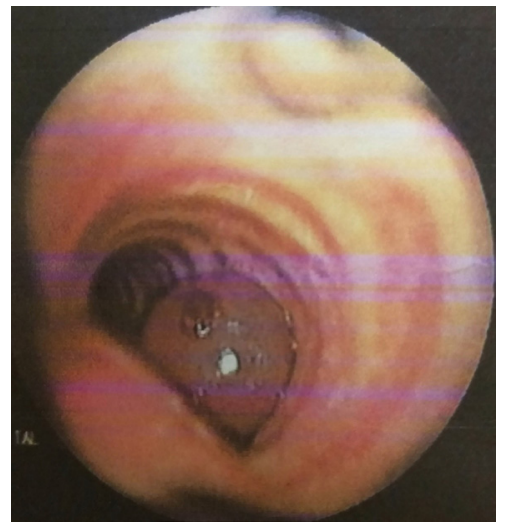

Figure 4. Endobronchial mass in right main bronchus without bleeding. 
cytological atypia and mitosis are existing.

In some reports, FEP is classified as an inflammatory mass (8). Several studies concluded that the shape of endobronchial FEP is lobulated with glossy surface $(7,9)$ or vegetating lobulated with long stalk (10), but in our case the surface of polyp was shiny and smooth.

In the literature of endobronchial FEP case reports, to the best our knowledge, no study has reported recurrent of these polyps yet, while our patient had recurrent endobronchial FEP.

\section{Conclusion}

Airway FEP is uncommon benign tumor that may obstruct airways and be misdiagnosed. Polyp resection with interventional bronchoscopy is a good procedure that results in efficacy treatment, but in some cases surgery may be required.

\section{Authors' contribution}

Case management and follow up by RS. Pathology report by NAM. Some parts of follow up and management by MN. Primary draft by $\mathrm{RS}$ and $\mathrm{MN}$. All authors read and signed the final paper.

Conflicts of interest

The authors declare that they have no competing interests.

\section{Ethical considerations}

Ethical issues (including plagiarism, data fabrication, double publication) have been completely observed by the authors. The patient gave informed consent for publication as a case report.
References

1. Stevic R, Milenkovic B. Tracheobronchial tumors. J Thorac Dis. 2016;8:3401. doi: 10.21037/jtd.2016.11.24.

2. Colak E, Ikinci A, Kucuk GO, Kesmer S, Yildirim K. Giant fibroepithelial polyp of the perineum: giant fibroepithelial polyp. Int J Surg Case Rep. 2015;17:126. doi: 10.1016/j. ijscr.2015.11.008.

3. Sindhwani G, Rawat J, Chauhan N, Kumari Mishra S. Tracheal polyp treated with endobronchial electrocautery. Indian J Chest Dis Allied Sci. 2012;54:123.

4. Desai P, Bernstein M. Fibroepithelial polyp of the bronchus: value of a cryobiopsy technique. Chest. 2018;154:875A. doi: 10.1016/j.chest.2018.08.788.

5. Labarca G, Caviedes I, Vial MR, Pires Y, Folch E, Majid A, et al. Airway fibroepithelial polyposis. Respir Med Case Rep. 2017;22:154-7. doi: 10.1016/j.rmcr.2017.08.005.

6. Schnader J, Harrell J, Mathur P, Joseph C, Koduri J, Kvale P. Clinical conference on management dilemmas: bronchiectasis and endobronchial polyps. Chest. 2002;121:637-43. doi: 10.1378/chest.121.2.637

7. Wartmann CT, Fernandez D, Flores RM. Fibroepithelial polyps: preoperative diagnosis may avoid thoracotomy. J Thorac Cardiovasc Surg. 2007;134:1080-1. doi: 10.1016/j. jtcvs.2007.05.032

8. Amin PB, Baciewicz F. Benign fibroepithelial polyp arising in the bronchus: a case report and review of the literature. Arch Surg. 2009;144:1081-3. doi: 10.1001/archsurg.2009.198.

9. Kang H, Kim TS, Han J, Kim H. Fibroepithelial polyp of the bronchus: CT and histopathologic findings. Korean J Radiol. 2012;13:355. doi: 10.3348/kjr.2012.13.3.355.

10. Dinçer Ib, Demir A, Akın $H$, Melek $H$, Altın S. A giant endobronchial inflammatory polyp. Ann Thorac Surg. 2005;80:2353-6. doi: 10.1016/j.athoracsur.2004.07.038. 